\title{
Effect of successive cyclic oral contraceptive pills on pregnancy outcome pretreatment of PCOS patient in ICSI
}

\author{
Hana Abd El Moneim Younes*, Yousef El Said Abo Shady*, Fayza A. Abdel Hakam*, Asmaa \\ Mohamed Awad Gomaa* \\ *Department of Obstetrics and Gynecology, Faculty of Medicine, Al-Azhar University \\ *Corresponding author: Asmaa Mohamed Awad Gomaa, Mobile: 00201129923985, E-Mail: z.yasmena@yahoo.com
}

\begin{abstract}
Introduction: Polycystic ovarian syndrome (PCOS) is a common endocrine disorder that primarily affects women of reproductive age, with prevalence rates ranging from $5 \%$ to $10 \%$.

Objective: To evaluate the role of oral contraceptive pills (OCP) pretreatment in the improvement of intra cytoplasmic sperm injection (ICSI) pregnancy outcome, in patient with PCOS.

Methodology: Started with 200 proved PCOS and after exclude of any patient with criteria rather than including criteria, so 60 proved PCOS women undergo ICSI were elected and divided into tow groups 30 patients for each. The $1^{\text {st }}$ group treated with COC (3-6monthes) before ICSI and the $2^{\text {nd }}$ group was a control group.

Results: Clinical pregnancy rate as a main outcome was significantly more frequent in the study group than in the control group. The LH mean as a secondary outcome was significantly more decreased in the study group than in the control group and the FSH/LH ratio before and after COC increasing ratio towards normalization.

Conclusion: Successive cyclic oral contraceptive pills for patients with PCOS as a pretreatment in ICSI can increase clinical pregnancy rates as a main outcome, improve the synchronism of follicular development, decrease the LH mean as a secondary outcome. And increase FSH/LH ratio before and after COC towards normalization.
\end{abstract}

Keywords: ICSI, Cyclic oral contraceptive pills, PCOS, Pregnancy outcome

\section{Introduction}

Polycystic ovarian syndrome (PCOS) is a common endocrine disorder that primarily affects women of reproductive age, with prevalence rates ranging from $5 \%$ to $10 \%{ }^{(\mathbf{1})}$.

One of the most common reproductive endocrine diseases that impact many young women worldwide is PCOS. This hormonal problem affects $4-18 \%$ of women of reproductive age exhibiting various symptoms, such as irregular menstruation, hirshutism, infertility and metabolic disorders ${ }^{(2)}$.

In 2013, a consensus panel established a controversial definition (the Rotterdam criteria) for PCOS, to include at least 2 of the following criteria: oligo- or anovulation (menses less than once every 35 days), Hyperandrogenism (laboratory-confirmed or clinical symptoms), or Polycystic ovaries on ultrasound. With exclusion of other conditions with similar signs such as androgen-secreting tumors, Cushing's syndrome, thyroid dysfunction and hyperprolactinemia ${ }^{(3)}$.

These symptoms in PCOS women are strongly correlated with overweight and obesity. Also, women with PCOS are likely to have metabolic disorders, such as abnormality of glucose and lipid metabolisms that are inevitably involved in diabetes mellitus and coronary artery diseases, respectively ${ }^{(\mathbf{4})}$.

Given these endocrine abnormalities, infertility is a common complication of PCOS. Studies have reported PCOS as the major cause of infertility in up to $20 \%$ of couples ${ }^{(5)}$.

Nearly eight decades following its original description, polycystic ovary syndrome (PCOS) remains a poorly understood entity. Various pathophysiologic mechanisms have been proposed; including intrinsic ovarian, adrenal, and hypothalamic- pituitary pathways, but none was shown to provide a complete explanation for the development of this condition. Much interest has occurred lately over the metabolic aspects of the syndrome, rendering insulin resistance with compensatory hyperinsulinemia the mechanism most widely studied today ${ }^{(6)}$.

Polycystic ovary syndrome remains to be a challenge to endocrinologists and gynecologists. As much as the pathogenesis is still not clear, so is the perfect way of treating 
this disease. Many medications are currently being used to treat PCOS, yet none of them is considered ideal. So, the question of a drug with better results and less side effects is still opened (7).

Metformin which is among the good treatment options for PCOS has a poor compliance record because of its many and distressing side effects mainly in form of nausea and diarrhea ${ }^{(8)}$.

The micronutrient chromium, which is gaining popularity as a dietary supplement to improve the actions of insulin under insulinresistant conditions, merits attention. The potential role of chromium in regulating blood sugar was first indicated in the late 195 os by Mertz and Schwarz. The 'essentiality' of chromium in human nutrition was suggested when it was found that chromium supplementation reversed glucose intolerance in hospitalized patients receiving long-term total parenteral nutrition ${ }^{(9)}$.

The combined oral contraceptive pills are considered the cornerstone in treatment of PCOS when fertility is not the main target of treatment $^{(\mathbf{1 0 )} \text {. }}$.

Combined oral contraceptives (COC) are the most often used treatment modality for polycystic ovary syndrome (PCOS). Undisputedly, COC suppress androgen production, thus ameliorating skin androgenic symptoms and improving menstrual dysfunction. On the other hand, there are still many unresolved issues concerning their metabolic effects. COC could decrease insulin sensitivity and deteriorate glucose tolerance, although the ofactors (especially obesity) and this need not be expressed in non-obese patients. It is probable that the impairment of glucose tolerance is reversible, as the incidence of diabetes is not increased in past COC users. The effects of COC on the lipid spectrum are dependent on the type of gestagen, but lipid levels usually remain within the reference limits. Combination therapy of $\mathrm{COC}$ with weight reduction or insulin sensitizers could further suppress androgen levels and improve metabolic parameters. The establishment of COC after laparoscopic ovarian drilling may further decrease androgen levels. The combination of $\mathrm{COC}$ and $\mathrm{GnRH}$ analogues is not superior to $\mathrm{COC}$ therapy alone. Prospective data about the influence of $\mathrm{COC}$ on the risk of diabetes mellitus, coronary artery disease and endometrial cancer in PCOS women are lacking ${ }^{(11)}$.

In vitro fertilization (IVF) is the most efficacious of existing fertility treatment strategies, albeit is also the most costly and invasive approach to infertility management. While IVF is clearly indicated for women with PCOS with concomitant tubal disease or severe male factor, this strategy should be considered for women failing to achieve successful pregnancy following multiple attempts at ovulation induction. Women with PCOS undergoing IVF treatment have similar pregnancy, miscarriage, and live-birth rates compared to those of non-PCOS patients, as evidenced by a large systematic review and meta-analysis of nine observational studies comparing 793 cycles of women with PCOS with 1116 cycles of matched control ${ }^{(\mathbf{1 2})}$.

\section{Aim of the study:}

To evaluate the role of oral contraceptive pills (OCP) pretreatment in the improvement of intra cytoplasmic sperm injection (ICSI) pregnancy outcome, in patient with polycystic ovarian syndrome (PCOS).

\section{Patient and Method}

Type of study: This study is comparative prospective study.

Setting and time: The study took place in the International Islamic Center for Population Studies and Research, Al-Azhar University from November 2016 till marc 2018. Sample size: Started with 200 proved polycystic ovarian syndrome (PCOS) and after exclusion of any patient with criteria rather than inclusion criteria, so 60 proved PCOS women undergo ICSI were elected and divided into tow groups 30 patient for each. The $1^{\text {st }}$ group treated with COC (3-6monthes) before ICSI and the $2^{\text {nd }}$ group was a control group.

\section{Ethical consideration:}

Approval was obtained by the Hospital Ethics Committee and informed consent was obtained before participation.

\section{Inclusion criteria:}

1. Patient with poly cystic ovaries by Rotterdam criteria of POCS ${ }^{(3)}$ which includes at least two of the following:-

a- Oligo- or anovulation.

b- Clinical and/or biochemical signs of hyperandrogenism.

c- Polycystic ovaries on transvaginal ultrasound.

2. Complaining of infertility (1ry or 2ry infertility). 
3. In child bearing period (20-35 years old).

4. Will undergo ICSI.

5. Consent from the patient about the nature of the study.

6. With no structural abnormality.

7. No endometriosis at any degree.

\section{Exclusion criteria:}

1. Refusal of participation in the study.

2. Any disorder of cardiovascular, pulmonary, hepatic, renal known from history or examination.

3. Patient unable to cost ICSI.

4. Patient passed the reproductive age (premenopausal).

5. Patient with combined causes of infertility.

6. Obesity with BMI $>35 \mathrm{Kg} / \mathrm{m}^{2}$.

7. Patient with: hypothyroidism and hyperprolactinemia.

Methods:

Patients were classified into two groups:

- Group A (study group): took (3-6) successive months of OCP and make hormonal profile (AMH / FSH /LH /E2 / TSH and prolactin) and Antral follicular count before and after the course of pretreatment before ICSI.

- Group B (control group): No pretreatment before ICSI.

Interventions:

A) Personal data: Full history taking.

- Patient's names, address, occupation, phone number were recorded.

B) Past history:

- History of medical disorders and operations.

- Family history of hormonal disorders or tumors.

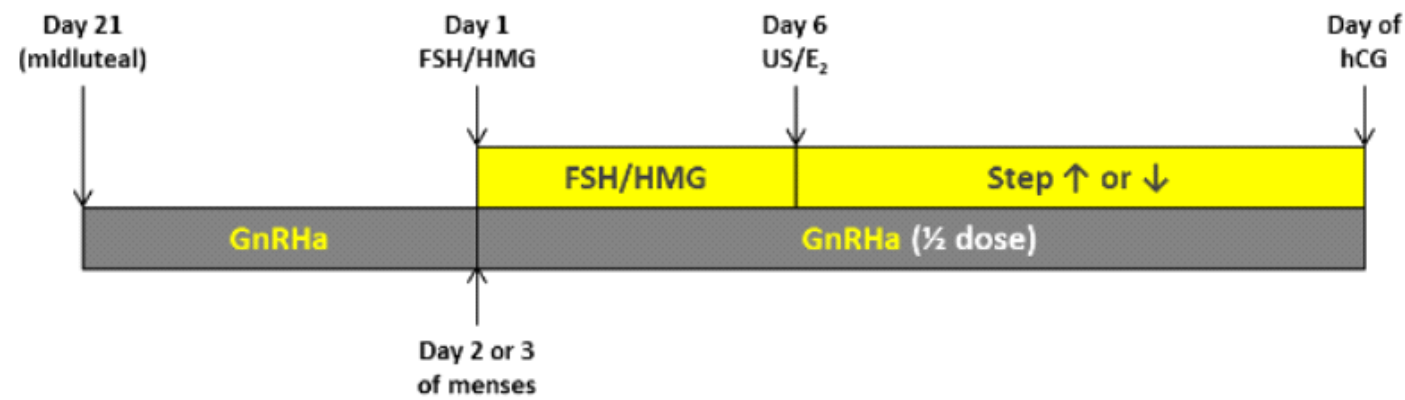

Ovarian stimulation was facilitated by the standard 'long protocol'. Briefly, pituitary down-regulation was achieved by $\mathrm{GnRH}$ agonist (Lucrin; Abbott.) in a dose of $1 \mathrm{mg}$ / day leuprolide acetate (Lucrin) started on the 21st day prior to menstruation for pituitary desensitization. When exogenous gonadotrophins were started on day 2 of
C) Obstetric history:

- Primary or secondary infertility.

D) Examination:

- General examination: Clinical examination including:

- Body weight measurement.

- Vital signs (heart rate, temperature, blood pressure and respiratory rate) color, hands and eyes.

- Abdomen and pelvic examination.

E) Laboratory investigation:

- Routine infertility investigations were performed in all couples. Semen analysis was evaluated according to the World Health Organization (WHO) criteria. ${ }^{(13)}$

- Hormonal profile (F.S.H /L.H /E2 / prolactin /T.S.H and A.M.H) on day 3 of a spontaneous or progesteroneinduced menstrual bleed before and after oral contraceptive pills.

- Pregnancy test after ICSI.

- Foliculomettry: measuring number and size of ovum before and after use of pills.

- Hystrosalpenjiography and hystrolaparoscopy were done for in need cases to exclude upper genital tract malformation, adhesion or endometriosis.

\section{Stimulation protocol for ICSI treatment:}

Recruitment all women undergoing ICSI cycle to Long-term protocol for induction of ovulation using $\mathrm{GnRH}$ agonist.

menstruation, the dose of leuprolide acetate was decreased to $0.5 \mathrm{mg} / \mathrm{day}$.

Ovarian stimulation was carried out with subcutaneous purified FSH The initial daily FSH dose was adjusted for age, ovarian volume and Antral follicle count, and varied between 150 to 225 I U. of HMG. Transvaginal ultrasound for folliculumetry was done every 3 - 
5 days and serum estradiol level is measured every 2-3 days by radioimmunoassay.

Ovulation was induced with $(5,000-$ 10000) IU human chorionic gonadotropin (HCG) when at least three follicles were $17 \mathrm{~mm}$ or more in maximum diameter.

Oocyte retrieval was performed by transvaginal follicular aspiration under vaginal ultrasound guidance 36-38 h after HCG administration. Oocytes were inseminated by ICSI and only ejaculated spermatozoa were microinjected.

Embryos were transferred on day 3 and as a rule, and when available, two embryos were transferred. However, transfer of three embryos was sometimes allowed in selected women in patients with poor embryo quality, after counseling the patients on the potential risks of multiple gestations.

The serum concentration of b-HCG was measured on day 14 after oocyte retrieval and, in the case of concentrations above $10 \mathrm{U} / \mathrm{L}$, which indicated pregnancy, an ultrasound scan was performed 3-4 weeks later to document viability. Positive serum pregnancy test without ultrasound signs of gestational sac was considered a biochemical pregnancy. Clinical pregnancy was diagnosed as presence of a gestational sac on transvaginal ultrasonography at 7 weeks' gestation or later.

Table (1): Demographic characteristics among the studied groups

\begin{tabular}{|c|c|c|c|}
\hline \multicolumn{2}{|c|}{ Variables } & Study $(\mathrm{N}=30)$ & Control $(\mathrm{N}=30)$ \\
\hline \multirow{3}{*}{$\begin{array}{c}\text { Age } \\
(\text { years })\end{array}$} & Mean & 26.93 & 25.43 \\
\hline & Range & $20-33$ & $20-33$ \\
\hline & SD & 3.73 & 3.92 \\
\hline \multirow{3}{*}{$\begin{array}{c}\text { BMI } \\
\left(\mathrm{kg} / \mathrm{m}^{2}\right)\end{array}$} & Mean & 27.71 & 27.61 \\
\hline & Range & $21-30.4$ & $20-30$ \\
\hline & SD & 2.93 & 3.07 \\
\hline \multirow[t]{3}{*}{ Parity } & Mean & 0.13 & 0.1 \\
\hline & Range & $0-2$ & $0-2$ \\
\hline & SD & 0.44 & 0.40 \\
\hline \multirow{3}{*}{$\overline{\text { AFC }}$} & Mean & 25.48 & 25.63 \\
\hline & Range & $21-30$ & $22-30$ \\
\hline & SD & 2.54 & 2.48 \\
\hline \multirow[t]{2}{*}{ Type of infertility $(n, \%)$} & Primary & 26 & 28 \\
\hline & Secondary & 4 & 2 \\
\hline \multirow{3}{*}{$\begin{array}{c}\text { Duration of infertility (n, } \\
\%)\end{array}$} & Mean & 5.20 & 4.5 \\
\hline & Range & $2-9$ & $1-12$ \\
\hline & SD & 1.80 & 2.66 \\
\hline
\end{tabular}

Table (2) shows the comparison between both groups regarding the demographic data and the duration of infertility indicate that there is no significant difference in age, BMI, parity, AFC, type and duration of
The two groups were compared as regard the following criteria:-

- Duration of stimulation.

- Total FSH dose/ cycle.

- Number of retrieved ova/ cycle.

- Number of embryos transfer and quality.

- Cycle pregnancy rate (clinical pregnancy is documented by fetal heart beating with ultrasound examination.).

\section{Statistical methods:}

The collected data were coded, tabulated, and statistically analyzed using SPSS statistics (Statistical Package for Social Sciences) software. Descriptive statistics were done for quantitative data as minimum\& maximum of the range as well as mean \pm SD (standard deviation) for quantitative normally distributed data, while it was done for qualitative data as number and percentage.

\section{Results}

\section{Demographic study of the patients}

Table (1) shows patient's age in years, Body mass index (BMI, $\mathrm{kg} / \mathrm{m}^{2}$ ), parity, AFC, Type of infertility, and Duration of infertility of the patients among the study group. 
Effect of successive cyclic oral contraceptive pills on pregnancy outcome pretreatment...

$(27.71 \pm 2.93,27.61 \pm 3.07) \mathrm{kg} / \mathrm{m} 2$,Parity of the patients varied between zero to 2 with a mean parity of $0.13 \pm 0.44,0.1 \pm 0.40$ Antral follicles count varied between 20 to 30 with a mean
$25.48 \pm 2.54,25.63 \pm 2.48$ and finally Duration of infertility (n, \%) varied between 1-12 with a mean $5.20 \pm 1.80,4.5 \pm 2.66$.

Table (2): Basal hormonal profile among the studied groups

\begin{tabular}{|c|c|c|c|}
\hline \multicolumn{2}{|c|}{ Variables } & \multirow{2}{*}{$\begin{array}{c}\text { Study }(\mathbf{N}=\mathbf{3 0}) \\
5.07\end{array}$} & \multirow{2}{*}{$\begin{array}{c}\text { Control }(\mathbf{N}=\mathbf{3 0}) \\
5.08\end{array}$} \\
\hline FSH & Mean & & \\
\hline & SD & 1.52 & 1.99 \\
\hline \multirow{2}{*}{$\begin{array}{l}\mathbf{L H} \\
(\mathrm{mIU} / \mathrm{mL})\end{array}$} & Mean & 4.22 & 4.92 \\
\hline & SD & 1.68 & 1.54 \\
\hline \multirow{2}{*}{$\begin{array}{l}\mathbf{E 2} \\
(\mathrm{pg} / \mathrm{mL})\end{array}$} & Mean & 45.75 & 50.39 \\
\hline & SD & 13.44 & 17.02 \\
\hline \multirow{2}{*}{$\begin{array}{l}\text { Prolactin } \\
\text { (ng/mL) }\end{array}$} & Mean & 18.06 & 18.8 \\
\hline & SD & 3.62 & 3.28 \\
\hline \multirow{2}{*}{$\begin{array}{l}\text { TSH } \\
(\mu \mathrm{IU} / \mathrm{mL})\end{array}$} & Mean & 2.37 & 2.57 \\
\hline & SD & 0.93 & 0.12 \\
\hline \multirow{2}{*}{$\begin{array}{l}\text { AMH } \\
\text { (ng/mL) }\end{array}$} & Mean & 3.5 & 3.4 \\
\hline & SD & 0.5 & 0.5 \\
\hline
\end{tabular}

No significant difference between study and control groups regarding basal FSH, LH, E2, prolactin, TSH, and AMH.

Table (3) HMG stimulation duration (days) among the studied groups

\begin{tabular}{|c|c|c|}
\hline HMG Duration & Study $(\mathbf{N}=\mathbf{3 0})$ & Control $(\mathbf{N}=\mathbf{3 0})$ \\
\hline Mean & 13.38 & 13.33 \\
\hline SD & 2.21 & 2.29 \\
\hline Range & $10-18$ & $9-19$ \\
\hline
\end{tabular}

No significant difference between study and control groups regarding HMG stimulation duration (days).

Table (4): The total dose of HMG in both groups:

\begin{tabular}{|c|c|c|}
\hline HMG total dose & Study $(\mathbf{N}=\mathbf{3 0})$ & Control $(\mathbf{N}=\mathbf{3 0})$ \\
\hline Mean & 2056.97 & 1914.533 \\
\hline SD & 668.3839 & 662.72 \\
\hline Range & $925-2625$ & $300-3150$ \\
\hline
\end{tabular}

No significant difference between study and control groups regarding the total dose of HMG in both groups.

Table (5): Oocyte number among the studied groups

\begin{tabular}{|c|c|c|}
\hline Oocyte number & Study $(\mathbf{N}=\mathbf{3 0})$ & Control $\mathbf{( N = 3 0 )}$ \\
\hline Mean & 11.6 & 11.47 \\
\hline SD & 4.17 & 4.61 \\
\hline Range & $1-20$ & $4-20$ \\
\hline
\end{tabular}

No significant difference among the control group and study group after stimulation Oocyte number.

Table (6): Oocyte retrieval among the studied groups

\begin{tabular}{|c|c|c|}
\hline Oocyte Retrieval & Study (N=30) & Control (N=30) \\
\hline Mean & 7.67 & 8.9 \\
\hline SD & 3.91 & 3.58 \\
\hline Range & $1-16$ & $2-16$ \\
\hline
\end{tabular}

No significant difference among the control group and study group after stimulation regarding Oocyte retrieval? 
Table (7): Fertilization among the studied groups

\begin{tabular}{|c|c|c|}
\hline Fertilization & Study (N=30) & Control (N=30) \\
\hline Mean & 3.57 & 3.23 \\
\hline SD & 2.16 & 1.52 \\
\hline Range & $1-8$ & $1-7$ \\
\hline
\end{tabular}

No significant difference among the control group and study group after stimulation regarding Fertilization among the studied groups.

Table (8): Embryo number among the studied groups

\begin{tabular}{|c|c|c|}
\hline Embryo number & Study (N=30) & Control $\mathbf{( N = 3 0 )}$ \\
\hline Mean & 3.57 & 3.23 \\
\hline SD & 2.16 & 1.52 \\
\hline Range & $1-8$ & $1-7$ \\
\hline
\end{tabular}

No significant difference among the control group and study group after stimulation regarding embryo number.

Table (9): Transferred embryo number among the studied groups

\begin{tabular}{|c|c|c|}
\hline Transferred embryo number & Study (N=30) & Control $(\mathbf{N}=\mathbf{3 0})$ \\
\hline Mean & 2.43 & 2.47 \\
\hline SD & 0.68 & 0.57 \\
\hline Range & $1-3$ & $1-3$ \\
\hline
\end{tabular}

No significant difference among the control group and study group after stimulation regarding transferred embryo number.

Table (10): Clinical Pregnancy among the studied groups

\begin{tabular}{|c|c|c|}
\hline Clinical Pregnancy & Study (N=30) & Control $(\mathbf{N}=\mathbf{3 0})$ \\
\hline Positive & $17(56.7 \%)$ & $13(43.3 \%)$ \\
\hline Negative & $13(43.3 \%)$ & $17(56.7 \%)$ \\
\hline Total & 30 & 30 \\
\hline
\end{tabular}

Table (10) shows that pregnancy test was done in 60 cases, positive clinical pregnancy was $17(56.7 \%)$ in patients with COC, while positive clinical pregnancy was 13 $(43.3 \%)$ in patients without COC.

Successive and cyclic oral contraceptive pill pretreatment improves IVF/ICSI outcomes of PCOS patients and ameliorates hyperandrogenism and Antral follicle excess ${ }^{(\mathbf{1 4})}$.

\section{Discussion}

The results of the present study revealed that all cases were homogenous in age, duration of infertility and BMI and showed that the clinical pregnancy rate which is the primary outcome of the study was more favorable in study group treated with COC (3-6 menthes) before ICSI than control group with no pretreatment as it represent only $(43.3 \%)$ in patients without COC group and $(56.7 \%)$ in study group with COC.

The clinical pregnancy was documented by fetal heart beating with transvaginal ultrasound. Thus, Pregnancy was more frequent among study group than among control group.
Successive and cyclic oral contraceptive pill pretreatment improves IVF/ICSI outcomes of PCOS patients and ameliorates hyperandrogenism and antral follicle excess ${ }^{(12)}$.

Certain patients have a tendency for high response to gonadotrophin therapy which is often not ameliorated with prior gonadotrophin-releasing hormone agonist (GnRHa) suppression. As a result, these patients are frequently cancelled and often experience ovarian hyperstimulation syndrome (OHSS) episodes during in-vitro fertilization (IVF)-embryo transfer cycles. Patients with polycystic ovarian syndrome (PCOS) have been noted to be particularly sensitive to exogenous gonadotrophin therapy. We have developed a protocol which is effective in improving IVF outcome in high responder patients, including those with PCOS. Oral contraceptive pills (OCP) are taken for 25 days followed by S.C. leuprolide acetate, $1 \mathrm{mg}$ /day, which is overlapped with the final 5 days of oral contraceptive administration ${ }^{(\mathbf{1 3 )}}$.

Low-dose gonadotrophin stimulation is then initiated on the third day of withdrawal 
bleeding in the form of either human menopausal gonadotrophins or purified urinary follicle-stimulating hormone at a dosage of 150 IU/day. Over a 5 year period, we reviewed our experience utilizing this dual method of suppression in 99 cycles obtained in 73 high responder patients. There were only 13 cancellations prior to embryo transfer (13.1\%). The clinical and ongoing pregnancy rates per initiated cycle were 46.5 and $40.4 \%$ respectively ${ }^{(14)}$.

Only eight patients experienced mildmoderate OHSS following treatment. For those patients who had undergone previous IVFembryo transfer cycles at our centre, significant improvements were noted in oocyte fertilization rates, embryo implantation rates and clinical/ongoing pregnancy rates with this protocol. Hormonal analyses revealed that the chief mechanism may be through an improved luteinizing hormone/follicle-stimulating hormone ratio following dual suppression. An additional feature of this dual method of suppression is significantly lower serum androgen concentrations, particularly dehydroepiandrosterone sulphate ${ }^{(14)}$.

But in contradiction to our results, the ongoing pregnancy rates per started cycle in the non-OCP group and OCP group were $27.8 \%$ and $12.7 \%(\mathrm{P}<0.05)$ respectively. The clinical pregnancy rate and embryo implantation rate were significantly reduced in OCP group (clinical pregnancy rate $16.5 \%$, implantation rate $11.2 \%$ ) as compared with non-OCP group $(32.2 \%$ and $19.9 \%, \mathrm{P}<0.05)$. The endometrium on hCG day was thicker in non-OCP group than OCP group $(9.5 \mathrm{~mm} \text { vs } 8.7 \mathrm{~mm}, \mathrm{P}<0.05)^{(14)}$.

Pretreatment with OCP, as compared with initiation of stimulation at Day 2 of cycle in patients treated with GnRH-a short protocol antagonist appears to be associated with a significant difference in ongoing pregnancy rates per started cycle. ${ }^{(\mathbf{1 5})}$

Also, in contradiction to our study, compared with the control group, OCP significantly reduced the formation of ovarian cyst $(\mathrm{P}<0.05)$, remarkably increased the duration of gonadotropin stimulation and consumption $(\mathrm{P}<0.01)$, and markedly raised the percentage of mature ova $(87.92 \%$ vs $92.85 \%, \quad \mathrm{P}<0.05)$. But no significant differences were observed between the two groups in the incidences of moderate and severe OHSS, number of retrieved oocytes, and rates of fertilization, miscarriage and clinical pregnancy ${ }^{(15)}$.

OCP for patients with PCOS can help to control the time of ovarian stimulation, improve the synchronism of follicular development, and increase the duration of gonadotropin stimulation and consumption, but cannot change the incidences of moderate and severe OHSS ${ }^{(15)}$.

\section{Conclusion}

Successive cyclic oral contraceptive pills for patients with PCOS as a pretreatment In ICSI can increase Clinical pregnancy rates as a main outcome, improve the synchronism of follicular development, decrease The LH mean as a secondary outcome. And increase FSH/LH ratio before and after $\mathrm{COC}$ towards normalization.

\section{References}

1- Franks S, Robinson S and Willis D (1996): Nutrition, insulin and polycystic ovary syndrome. Reviews of Reproduction, 1: 47-53.

2- $\quad$ Moran LJ, Hutchison SK, Norman RJ et al. (2011): Lifestyle changes in women with polycystic ovary syndrome. Cochrane Database Syst. Rev., (2):CD007506.

3- Rotterdam ESHRE/ASRM-Sponsored PCOS Consensus Workshop Group (2004): Revised 2003 consensus on diagnostic criteria and long-term health risks related to polycystic ovary syndrome. Fertil. Steril., 81(1):19-25.

4- Traub ML (2011): Assessing and treating insulin resistance in women with polycystic ovarian syndrome. World J. Diabetes, 2: 33-40.

5- Broekmans F, Knauff E, Valkenburg O et al. (2006): PCOS according to the Rotterdam consensus criteria: change in prevalence among WHO-II anovulation and association with metabolic factors. BJOG., 113:1210-1217.

6- Diamanti-Kandarakis E and Papavassiliou AG (2006): Molecular mechanisms of insulin resistance in polycystic ovary syndrome. Trends. Mol. Med.,12(7):324-32.

7- Teede H, Deeks $A$ and Moran $L$ (2010): Polycystic ovary syndrome: a complex condition with psychological, reproductive and metabolic 
manifestations that impacts on health across the lifespan. B.M.C. Medicine, 8:

41.

8- Tang T, Lord JM, Norman RJ et al. (2012): Insulin-sensitizing drugs (metformin, rosiglitazone, pioglitaazone, D-chiro-inositol) for women with polycystic ovary syndrome, oligo amenorrhoea and subfertility. Cochrane Dtabase. Syst. Rev., 16(5): CD003053.

9- Jeejeebhoy KN, Chu RC, Marliss EB et al. (1977): Chromium deficiency, glucose intolerance, and neuropathy reversed by chromium supplementation, in a patient receiving long-term total parenteral nutrition. Am. J. Clin. Nutr., 30(4):531-8.

10- Sirmans $S$ and Pate $K$ (2014): Epidemiology, diagnosis, and management of polycystic ovary syndrome. Clin. Epidemiol., 6: 1-13.

11- Vrbíková J and Cibula D (2005): Combined oral contraceptives in the treatment of polycystic ovary syndrome. Hum. Reprod. Update, 11(3):277-91.
12- Practice Committee of the American Society for Reproductive Medicine (2008): Ovarian hyperstimulation syndrome. Fertil. Steril., 90(5): 178-83.

13- Rowe PJ, Comhaire FH, Hargreave TB et al. (1993): WHO Manual for the Standardized Investigation of the Infertile Couple. Cambridge University Press, Cambridge, UK. https://biblio.ugent.be/publication/2345 29

14- Pan JX, Liu Y, Ke ZH et al. (2015): Successive and cyclic oral contraceptive pill pretreatment improves IVF/ICSI outcomes of PCOS patients and ameliorates hyperandrogenism and antral follicle excess. Gynecol. Endocrinol., 31(4):332-336.

15- Yang S, Chen XN, Qiao J et al. (2012): Comparison of GnRH antagonist fixed protocol and $\mathrm{GnRH}$ agonists long protocol in infertile patients with normal ovarian reserve function in their first in vitro fertilization-embryo transfer cycle. Zhonghua. Fu. Chan. Ke. Za. Zhi., 47(4):245-9. 